\title{
Crystal fields of dilute Tb, Dy, Ho, or Er in Lu obtained by magnetization measurements
}

\section{Touborg, P.; Høg, J.}

\section{Published in:}

Physical Review B

Link to article, DOI:

10.1103/PhysRevB.11.2660

Publication date:

1975

\section{Document Version}

Publisher's PDF, also known as Version of record

Link back to DTU Orbit

Citation (APA):

Touborg, P., \& Høg, J. (1975). Crystal fields of dilute Tb, Dy, Ho, or Er in Lu obtained by magnetization measurements. Physical Review B, 11(7), 2660-2667. https://doi.org/10.1103/PhysRevB.11.2660

\section{General rights}

Copyright and moral rights for the publications made accessible in the public portal are retained by the authors and/or other copyright owners and it is a condition of accessing publications that users recognise and abide by the legal requirements associated with these rights.

- Users may download and print one copy of any publication from the public portal for the purpose of private study or research.

- You may not further distribute the material or use it for any profit-making activity or commercial gain

- You may freely distribute the URL identifying the publication in the public portal 


\title{
Crystal fields of dilute $\mathrm{Tb}$, Dy, Ho, or Er in Lu obtained by magnetization measurements
}

\author{
P. Touborg* and J.Høg \\ Department of Electrophysics, Technical University, 2800 Lyngby, Denmark
}

(Received 16 September 1974)

\begin{abstract}
Magnetization measurements are reported on single crystals of dilute $\mathrm{Tb}$, Dy, Ho, or Er in Lu. These measurements were performed in the temperature range $1.5-100 \mathrm{~K}$ and field range 0-6 $\mathrm{T}$ and include measurements of initial susceptibility, isothermal and isofield magnetization, and basal-plane anisotropy. The results show features similar to the corresponding $\mathrm{Y}-R$ alloys, where $R$ is a rare earth. Crystal-field and molecular-field parameters could be unabiguously deduced from the experimental data. The effects of crystal-field level broadening were investigated and demonstrated for Ho. Comparison of the $\mathrm{Y}-R$ and Lu- $R$ results makes possible an estimate of the crystal-field parameters in the pure-rare-earth metals.
\end{abstract}

\section{INTRODUCTION}

In two previous papers $\mathrm{s}^{1,2}$ the crystal-field and exchange parameters of dilute $\mathrm{Tb}$, Dy, or $\mathrm{Er}$ in $\mathrm{Y}$ have been reported. The parameters which were obtained by fits to the inverse initial susceptibilies have been checked by different kinds of measurements. These are low-field ${ }^{1,2}$ (to $6 \mathrm{~T}$ ) and highfield $^{3}$ (to $37 \mathrm{~T}$ ) magnetization measurements, basal-plane-anisotropy measurements, ${ }^{2}$ magnetization measurements on samples of different concentrations, ${ }^{2}$ and an inelastic-neutron-diffraction experiment. ${ }^{4}$ The crystal-field parameters were found to depend unsystematically on the rareearth solute, and the exchange parameters turned out to be highly anisotropic.

The variation of the crystal-field parameters across the rare-earth series demonstrates the necessity of a first-principles calculation of the crystal field based on the electronic band structure. Such calculations are at present only at a preliminary stage. ${ }^{5}$ The presence of anisotropic exchange interactions in the pure-rare-earth metals, which has recently been demonstrated, ${ }^{6}$ implies that no reliable experimental values of the crystal-field parameters exist for these metals.

The crystal-field parameters for rare earths diluted in $\mathrm{Y}$ are expected to give a good measure of the crystal fields in the pure-rare-earth metals. However, deviations may be expected owing to dif ferences in lattice parameters and electronic band structures. In Table $I$ is shown how the lattice constants and the $d$-conduction-bandwidths ${ }^{7}$ of $\mathrm{Tb}$, Dy, Ho, and Er are close to and bounded by those of $\mathrm{Y}$ and $\mathrm{Lu}$. Measurements of the crystal fields in both $\mathrm{Y}$ and the nonmagnetic heavy-rare-earth metal $\mathrm{Lu}$ may therefore be expected to offer an excellent means of obtaining estimates of the crystal fields in the pure-rare-earth metals.
In this paper we present the results of such an experiment. Magnetization measurements on single crystals of dilute $\mathrm{Tb}$, Dy, Ho, or Er in $\mathrm{Lu}$ are reported, with deduced crystal-field and exchange parameters. We have performed measurements of initial susceptibility, isofield, and isothermal magnetization, and also of the basalplane anisotropy.

\section{EXPERIMENTAL PROCEDURE}

Using sublimed materials of $99.9 \%$ purity, single crystals of pure $\mathrm{Lu}$ and $\mathrm{Lu}-R$ alloys where $R$ is a rare earth, were grown by the recrystallization technique described in Ref. 8. The largest single crystal of pure Lu grown by this method had a weight of $10 \mathrm{~g}$. During these experiments a transition in $\mathrm{Lu}$ at $(1530 \pm 30)^{\circ} \mathrm{C}$ from the low-temperature hcp structure to a high-temperature allotropic form was detected. To our knowledge no such transition has been published earlier. In order to get an accurate measure of the Lu background in the alloys, pure Lu samples were cut from Lu single crystals and measured. The same Lu single crystals were remelted afterwards and used as host material in the alloys. Samples in the form of $2.5-\mathrm{mm}$ spheres were prepared of the following alloys: $\mathrm{Lu}-0.634 \% \mathrm{~Tb}, \mathrm{Lu}-0.133 \% \mathrm{~Tb}$, $\mathrm{Lu}-0.481 \% \mathrm{Dy}, \mathrm{Lu}-0.978 \% \mathrm{Ho}$, and $\mathrm{Lu}-0.538 \% \mathrm{Er}$ (all concentrations are given in atomic percent).

The measurements were performed using three different magnetometers, which have all been described earlier. A Faraday magnetometer ${ }^{1}$ was used for measuring initial susceptibilities, a $5-\mathrm{Hz}$ vibrating-sample magnetomete ${ }^{1}$ was used for measuring isothermal and isofield magnetization curves at higher fields and a $169-\mathrm{Hz}$ vibratingsample anisotropy meter ${ }^{2,9}$ was used for measuring the basal-plane anisotropy. In the latter instrument the horizontal component of the mag- 
TABLE I. Electronic properties and lattice parameters for six hcp metals.

\begin{tabular}{llccccc}
\hline \hline Metal & Configuration & $\begin{array}{c}d \text {-bandwidth } \\
(\text { Ry) })^{2}\end{array}$ & Ground state & $a(\AA)$ & $c(\AA)$ & $c / a$ \\
\hline $\mathrm{Y}$ & $\mathrm{Kr}+4 d^{1} 5 s^{2}$ & 0.48 & $\ldots$ & 3.650 & 5.741 & 1.573 \\
$\mathrm{~Tb}$ & $\mathrm{Xe}+5 d^{1} 6 s^{2} 4 f^{8}$ & 0.59 & ${ }^{7} F_{6}$ & 3.604 & 5.698 & 1.581 \\
$\mathrm{Dy}$ & $\mathrm{Xe}+5 d^{1} 6 s^{2} 4 f^{9}$ & $\ldots$ & ${ }^{9} H_{15 / 2}$ & 3.593 & 5.655 & 1.574 \\
$\mathrm{Ho}$ & $\mathrm{Xe}+5 d^{1} 6 s^{2} 4 f^{10}$ & $\ldots$ & ${ }^{5} I_{8}$ & 3.578 & 5.626 & 1.572 \\
$\mathrm{Er}$ & $\mathrm{Xe}+5 d^{1} 6 s^{2} 4 f^{11}$ & $\ldots$ & ${ }^{4} I_{15 / 2}$ & 3.560 & 5.595 & 1.572 \\
$\mathrm{Lu}$ & $\mathrm{Xe}+5 d^{1} 6 s^{2} 4 f^{14}$ & 0.61 & $\ldots$ & 3.505 & 5.553 & 1.584 \\
\hline \hline
\end{tabular}

${ }^{\mathrm{a}}$ Reference 7 .

netic moment, $M_{\perp}$, perpendicular to the horizontal external field, was measured as a function of crystal rotation angle $\phi$ about the vertical $c$ axis. $M_{\perp}(\phi)$, which was an almost pure sixth-order harmonic, was Fourier-analyzed.

\section{THEORETICAL PROCEDURE}

The appropriate Hamiltonian for interpretation of this experiment is

$$
\begin{aligned}
\mathcal{H C}= & \left(B_{20} O_{20}+B_{40} O_{40}+B_{60} O_{60}+B_{66} O_{66}\right) \\
& +g_{J} \mu_{B} \vec{J} \cdot(\overrightarrow{\mathrm{H}}+\hat{\lambda} \cdot \overrightarrow{\mathrm{M}}),
\end{aligned}
$$

where the terms in the first set of parentheses constitute the crystal-field operator, and the second term is the effective Zeeman operator, which includes the relatively small exchange interaction in the molecular-field approximation. The $O_{l m}$ 's are the Stevens operators, ${ }^{10}$ the $B_{l m}$ 's are the crystal-field parameters, $g_{J}$ is the Landé factor, $\mu_{B}$ is the Bohr magneton, $\vec{J}$ is the total angular momentum operator, $\overrightarrow{\mathrm{H}}$ is the internal field, $\hat{\lambda}$ the molecular-field tensor (containing two independent parameters $\lambda_{\perp}$ and $\lambda_{\|}$), and $\vec{M}$ is the magnetization per rare-earth atom. The $B_{l m}$ 's, $\lambda_{\perp}$, $\lambda_{\|}$, and the actual solute concentration $c$ were obtained by simultaneous fits to the inverse initial susceptibilities in the $c$ and $b$ directions. Owing to expected short-range-order effects at low temperatures, several fits were performed in which the lowest temperature data $\left(T<T_{0}\right)$ were disregarded. The errors in the parameters were estimated from their variation with $T_{0}$ in the temperature range 3-8 $\mathrm{K}$. These errors are one order of magnitude larger than those obtained from the sharpness of the minimum in the least-squares deviation. The fitting procedure used is described in more detail in Ref. 1. Isofield, isothermal, and basal-plane anisotropy curves were used to check the reliability of the deduced parameters and to reveal ordering effects, etc. In the case of $\mathrm{Tb}$ the measurements of $M_{\perp}(H, T, \phi)$ had to be included in the fit.

A recent neutron diffraction experiment ${ }^{4}$

Y-2\% Er has demonstrated broadening of the crystal-field levels with a full width at half-maximum (FWHM) of approximately $11 \mathrm{~K}$ for the first excited doublet state. A calculation based on isotropic nearest-neighbor exchange using the exchange constants for pure Er gives a linewidth of this order of magnitude. ${ }^{11}$ Interactions with the conduction electrons, nonequivalent sites in the disordered alloy, and interactions between the crystal field and the phonon modes may also contribute to broadening of the crystal-field levels. The effects of level broadening on the initial sus ceptibility were estimated from a single-ion calculation assuming the following distribution function for the energy levels at zero applied field:

$$
f\left(E_{1}, \ldots, E_{2 J+1}\right)=\sum_{i=1}^{2 J+1}\left(2 \pi \Gamma_{i}^{2}\right)^{-1 / 2} e^{-\left(E_{i}-E_{i}^{0}\right)^{2} / 2 \Gamma_{i}^{2}} .
$$

$E_{i}^{0}$ are the energy levels calculated from the field parameters and $\Gamma_{i}$ are the widths [the FWHM is $\left.(8 \ln 2)^{1 / 2} \Gamma\right]$ of the levels $i$ at $H=0$. We have as sumed in the calculations that the eigenfunctions for all $E_{i}($ at $H=0)$ are the same, $\psi_{i}^{0}$, as calculated for $E_{i}^{0}$. This is a good approximation for small level widths and also for larger widths if the matrix elements of the interactions giving rise to broadening are small between the $\psi_{i}^{0}$ states. With FWHM's as large as $7 \mathrm{~K}$ for these dilute alloys, compared to $11 \mathrm{~K}$ observed for $\mathrm{Y}-2 \% \mathrm{Er}$, the calculations showed that the effects of level broadening on the susceptibilities were only essential be low $5 \mathrm{~K}$.

\section{RESULTS}

In this section experimental data and theoretical curves are presented. In all cases the contribution from the Lu host to the total magnetic moment of the sample has been subtracted, giving the moment of the rare-earth impurity separately. In 


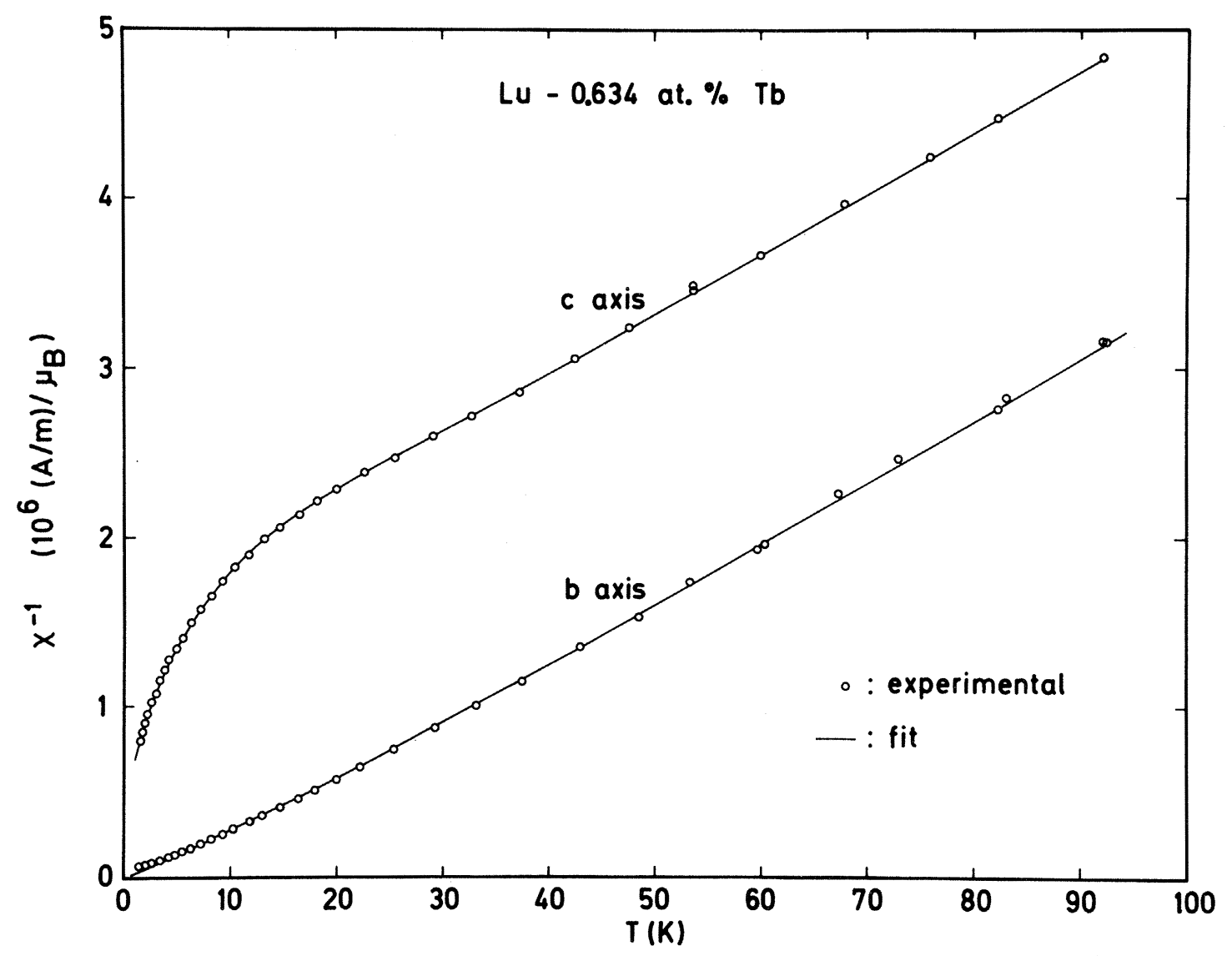

FIG. 1. Reciprocal susceptibility for $\mathrm{Lu}-0.634$ at. $\% \mathrm{~Tb}\left(H=1.35 \times 10^{5} \mathrm{~A} / \mathrm{m}\right)$. The solid curves are theoretical fits.

the following we present results for each rareearth solute individually.

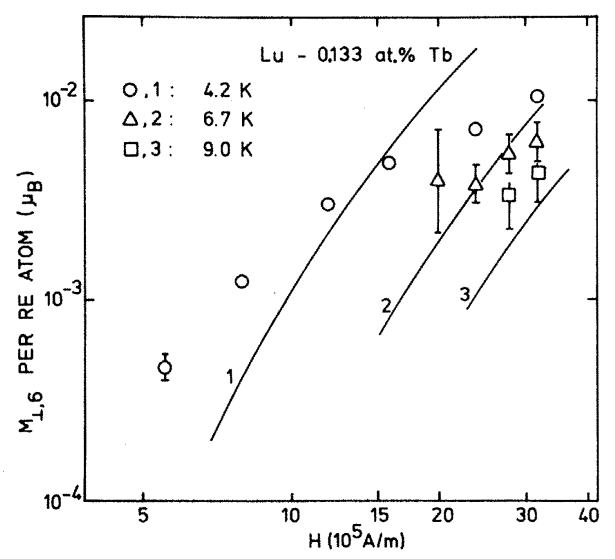

FIG. 2. Sixth-order harmonic of the perpendicular basal-plane magnetization vs field and temperature for $\mathrm{Lu}-0.133$ at. $\% \mathrm{~Tb}$. The solid curves are theoretical fits.
$\mathrm{Tb}(J=6)$

The parameters for $\mathrm{Tb}$ were obtained by simultaneous fits to data of the reciprocal initial sus ceptibilities (Fig. 1) and the basal-plane anisotropy (Fig. 2). It was necessary to include the latter measurements due to the smooth behavior shown by the former. Of the four rare earths studied in this work, Tb exhibits ordering effects at the highest temperatures, as is the case for the pure metals. It was necessary to prepare a more dilute alloy for the basal-plane-anisotropy measurements used in the fit since these measurements were very sensitive to ordering (measurements on varying concentrations of $\mathrm{Y}-R$ alloys have shown that the crystal-field parameters are independent of the concentration at low concentration). Even for the $\mathrm{Lu}-0.133 \% \mathrm{~Tb}$, the effects of ordering can be seen at $4.2 \mathrm{~K}$ (Fig. 2). Also the isothermal and isofield magnetization data (Figs. 3 and 4) show large deviations from theoretical curves at low temperatures. This is attributed to combined effects of ordering, level broadening (which is very sensitive to ordering), and conduction-electron polarization. 


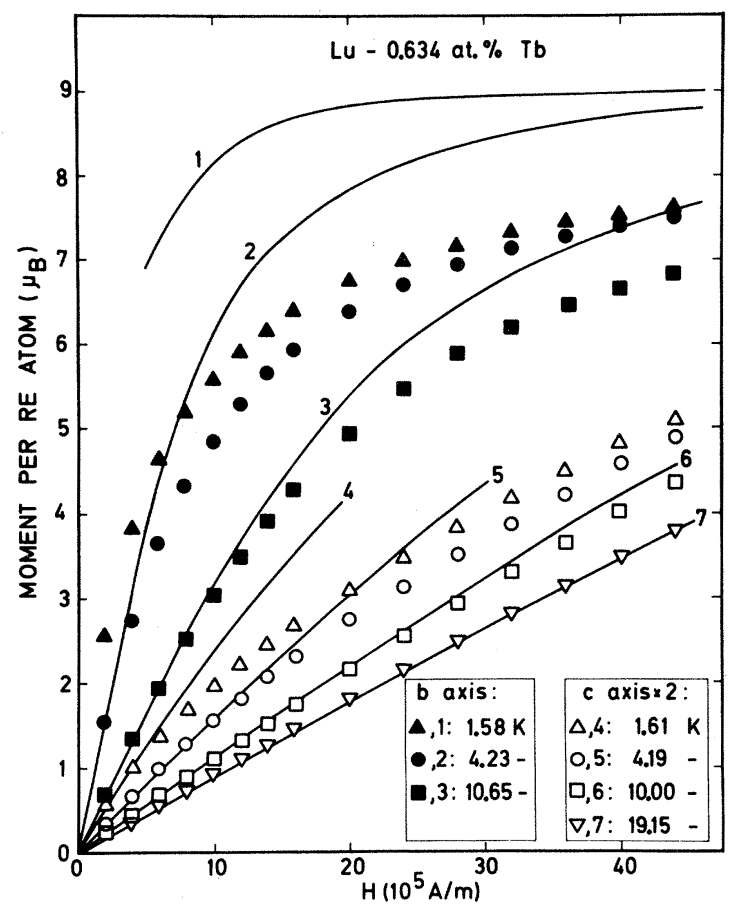

FIG. 3. Isotherms for $\mathrm{Lu}-0.634$ at.\% $\mathrm{Tb}$. The solid curves are calculated.

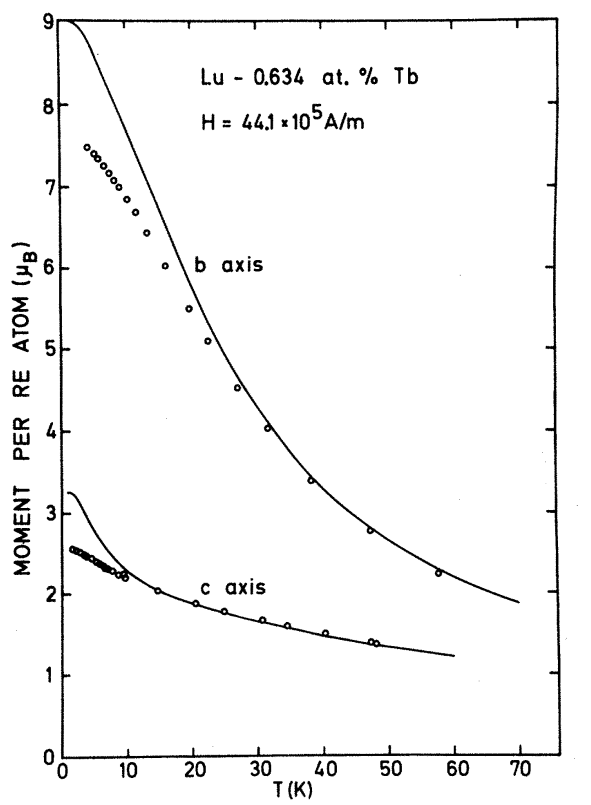

FIG. 4. Isofield magnetization curves for $\mathrm{Lu}-0.634$ at.\% $\mathrm{Tb}$. The solid curves are calculated.

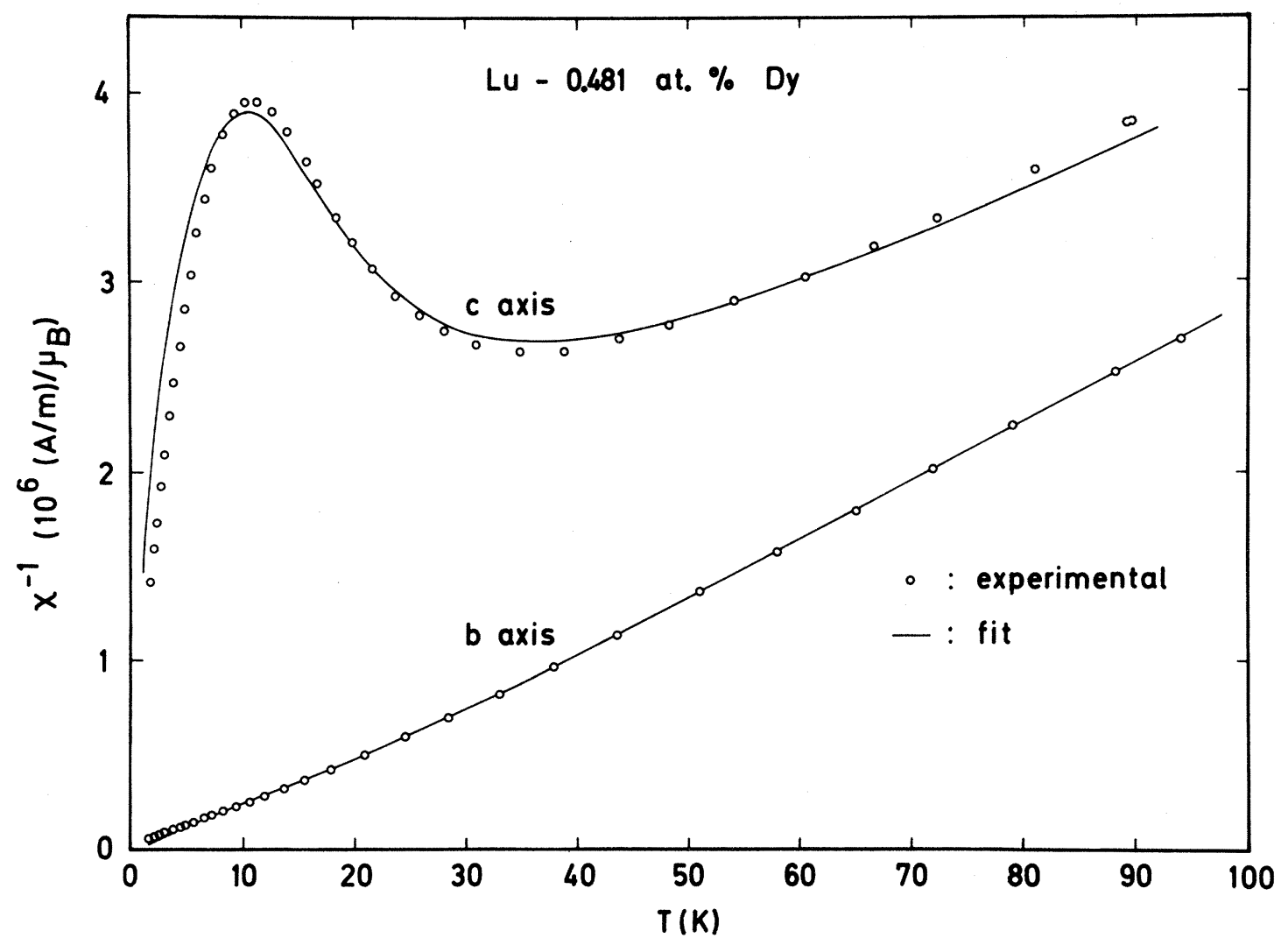

FIG. 5. Reciprocal susceptibility for $\mathrm{Lu}-0.481$ at. $\% \mathrm{Dy}$. $\left(H=1.35 \times 10^{5} \mathrm{~A} / \mathrm{m}\right)$. The solid curves are theoretical fits. 


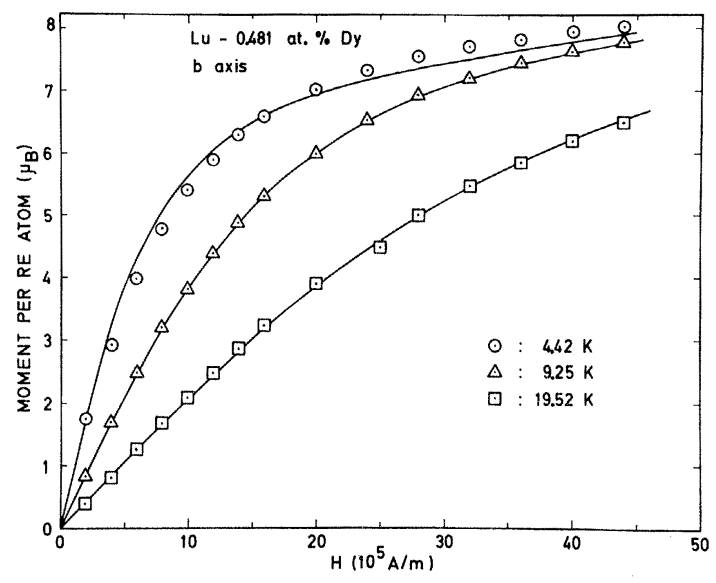

FIG. 6. Isotherms in the $b$ direction for $\mathrm{Lu}-0.481$ at. \% Dy. The solid curves are calculated.

Owing to these effects being most severe in $\mathrm{Tb}$ and to the smooth behavior of the susceptibility curves, the parameters for $\mathrm{Tb}$ are determined with relatively large inaccuracies.

$$
\text { Dy }\left(J=\frac{15}{2}\right)
$$

The inverse-initial-susceptibility data for Dy (Fig. 5) show characteristic features permitting an unambiguous determination of all parameters. Like $\mathrm{Lu}-0.634 \% \mathrm{~Tb}$, the $\mathrm{Lu}-0.481 \%$ Dy shows incipient ordering at the lowest temperatures (Figs. 1 and 5 ). The isotherms in the $b$ direction above $4.2 \mathrm{~K}$ show good consistency between theory and experiment (Fig. 6). This is contrary to the isotherms in the $c$ direction (Fig. 7), where deviations up to temperatures as high as $15 \mathrm{~K}$ are seen. These deviations, which are also illustrated in the isofield

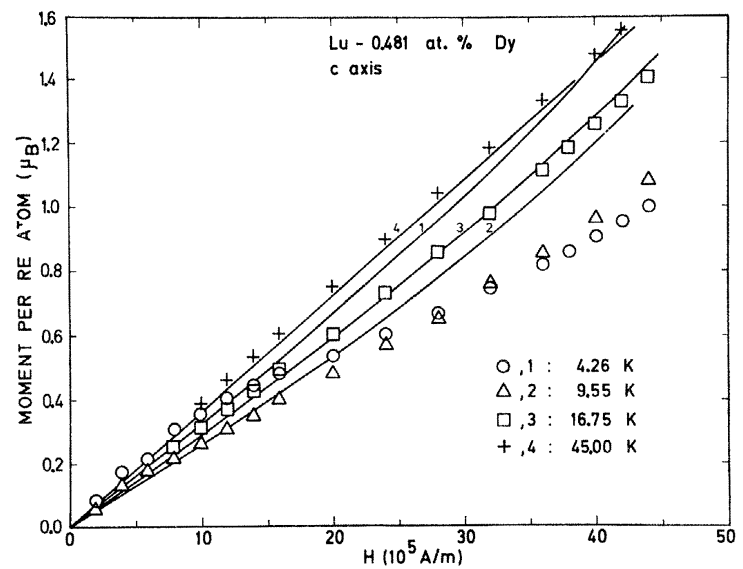

FIG. 7. Isotherms in the $c$ direction for $\mathrm{Lu}-0.481$ at. \% Dy. The solid curves are calculated.

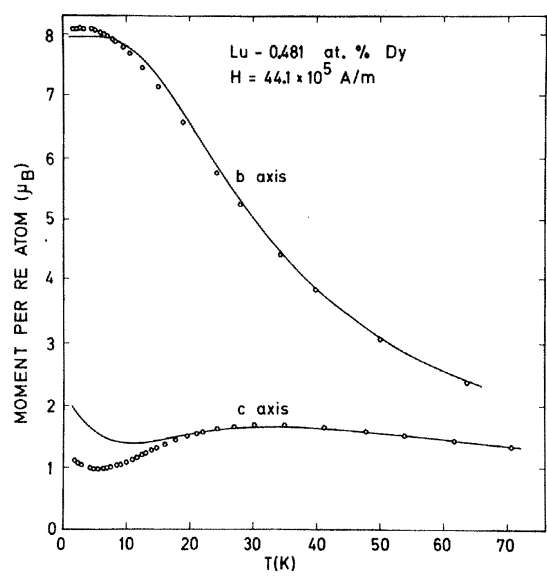

FIG. 8. Isofield magnetization curves for $\mathrm{Lu}-0.481$ at. $\%$ Dy. The solid curves are calculated.

magnetization curves (Fig. 8), are similar to those in Y-Dy alloys. ${ }^{1.2}$ Also, the basal-plane-anisotropy curves (Fig. 9) show similar characteristic features to those seen for the Y-Dy alloys and for pure Dy (Ref. 12) in the helical phase. For pure Dy these characteristics were attributed to the formation of a fan structure. ${ }^{12}$

$$
\text { Ho }(J=8)
$$

For Ho, only measurements of the initial susceptibilities were performed (Fig. 10). These provided a unique and accurate fit with $T_{0}=6 \mathrm{~K}$. The calculated reciprocal $c$-axis susceptibility increases sharply and deviates from the experimental data below $5 \mathrm{~K}$ (the dashed curve in Fig. 10). The ground state is the singlet $2^{-1 / 2}(|8,3\rangle-|8,-3\rangle)$

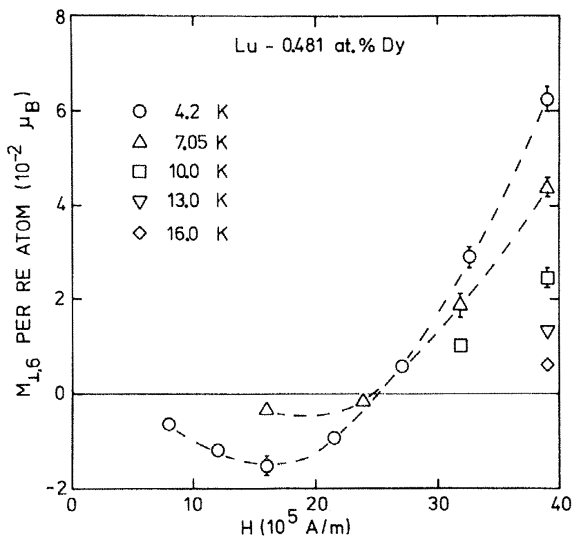

FIG. 9. Sixth-order harmonic of the perpendicular basal-plane magnetization vs field and temperature for $\mathrm{Lu}-0.481$ at.\% Dy. The dashed curves are drawn by hand. 


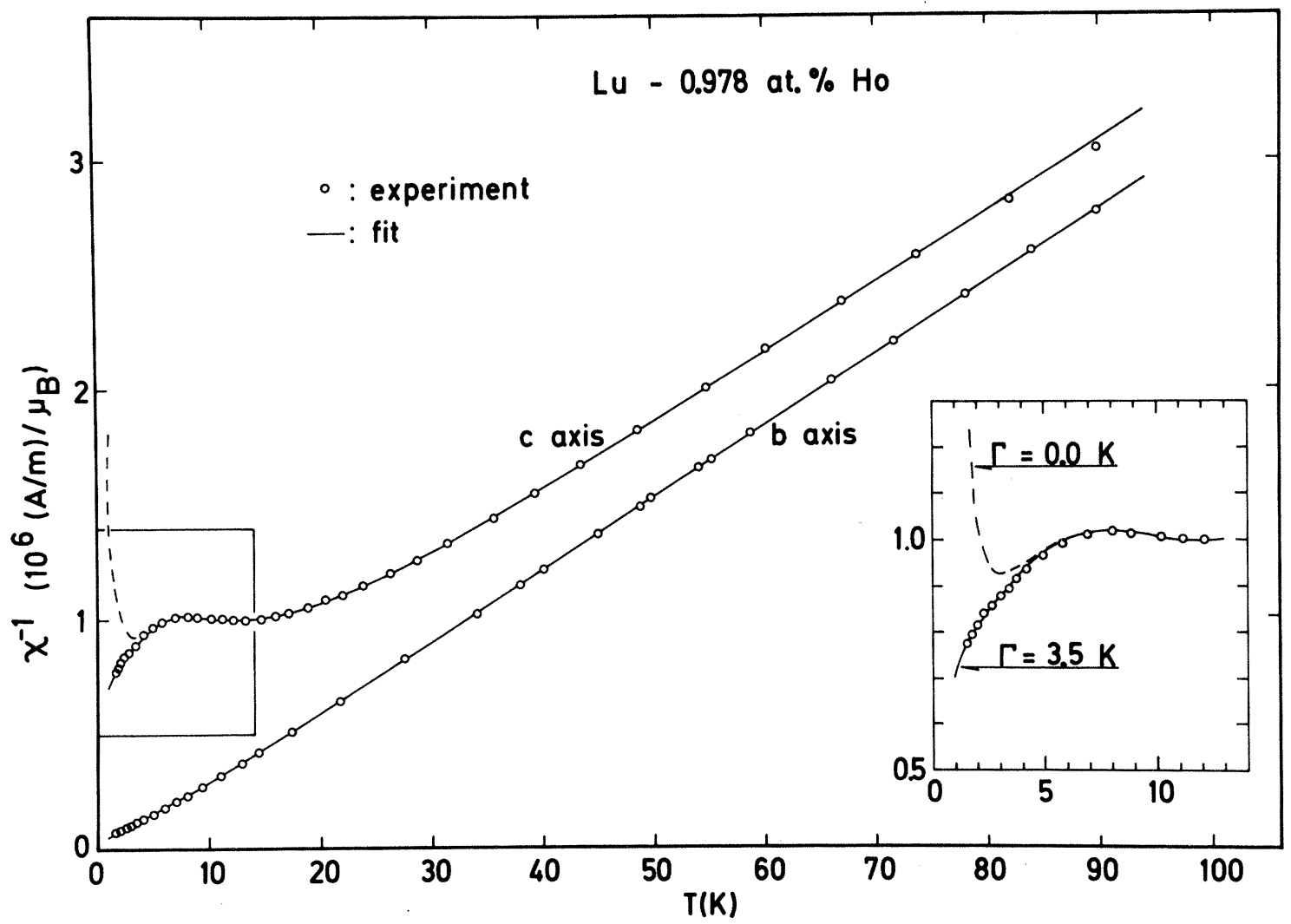

FIG. 10. Reciprocal susceptibility for $\mathrm{Lu}-0.978$ at. $\% \mathrm{Ho}\left(H=1.35 \times 10^{5} \mathrm{~A} / \mathrm{m}\right)$. The dashed curves are calculated neglecting level broadenings. The solid curves are calculated assuming a broadening of the first excited doublet as described in the text.

which is separated by $4.6 \mathrm{~K}$ from the first excited state $0.850|8, \pm 4\rangle-0.520|8, \mp 2\rangle+0.083|8, \mp 8\rangle$. The susceptibilities were calculated assuming a level broadening of $\Gamma=0$ for the singlet ground state, $\Gamma=3.5 \mathrm{~K}$ for the first excited doublet, and $\Gamma=0$ for all higher states whose broadenings have little effect. These susceptibilities account excellently for the experimental data also at low temperatures as can be seen from the solid curve in Fig. 10. If the level broadening originates in exchange inter actions, singlet states will have $\Gamma=0$ to first order in the molecular field.

$$
\operatorname{Er}\left(J=\frac{15}{2}\right)
$$

The reciprocal initial susceptibilities for $\mathrm{Er}$ (Fig. 11) are less characteristic, but could be uniquely fitted. The isothermal (Fig. 12) and isofield (Fig. 13) magnetization curves provide a good check of the parameters obtained.

\section{DISCUSSION}

The magnetization measurements on dilute $\mathrm{Lu}-R$ alloys show the same characteristic features as for the corresponding $\mathrm{Y}-R$ measurements, and the same excellent fits at higher temperatures are obtained. At lower temperatures the Lu-R show more tendency to ordering, which also may result in a larger degree of level broadening. A deviation at low temperatures between the experimental and theoretical susceptibility of Lu-Ho may be accounted for as arising from the effects of level broadening. This is illustrated in the inset in Fig. 10. Calculations for $\mathrm{Lu}-\mathrm{Tb}, \mathrm{Lu}-\mathrm{Er}$, and Lu-Dy showed qualitatively that the effect of broadening was significant only at low temperatures, where also ordering and conduction-electron polarization are important. The values of the fitted crystalfield parameters are not affected by these effects as the low-temperature data have been disregarded in the fits.

In Table II the crystal-field and the molecularfield parameters are summarized. The exchange parameters are highly anisotropic and compare well with those for Y-R $R^{2}$ except $\lambda_{\perp}$ for Er, which is smaller in Lu-Er.

Table III summarizes the crystal-field parameters divided by the corresponding Stevens fac- 


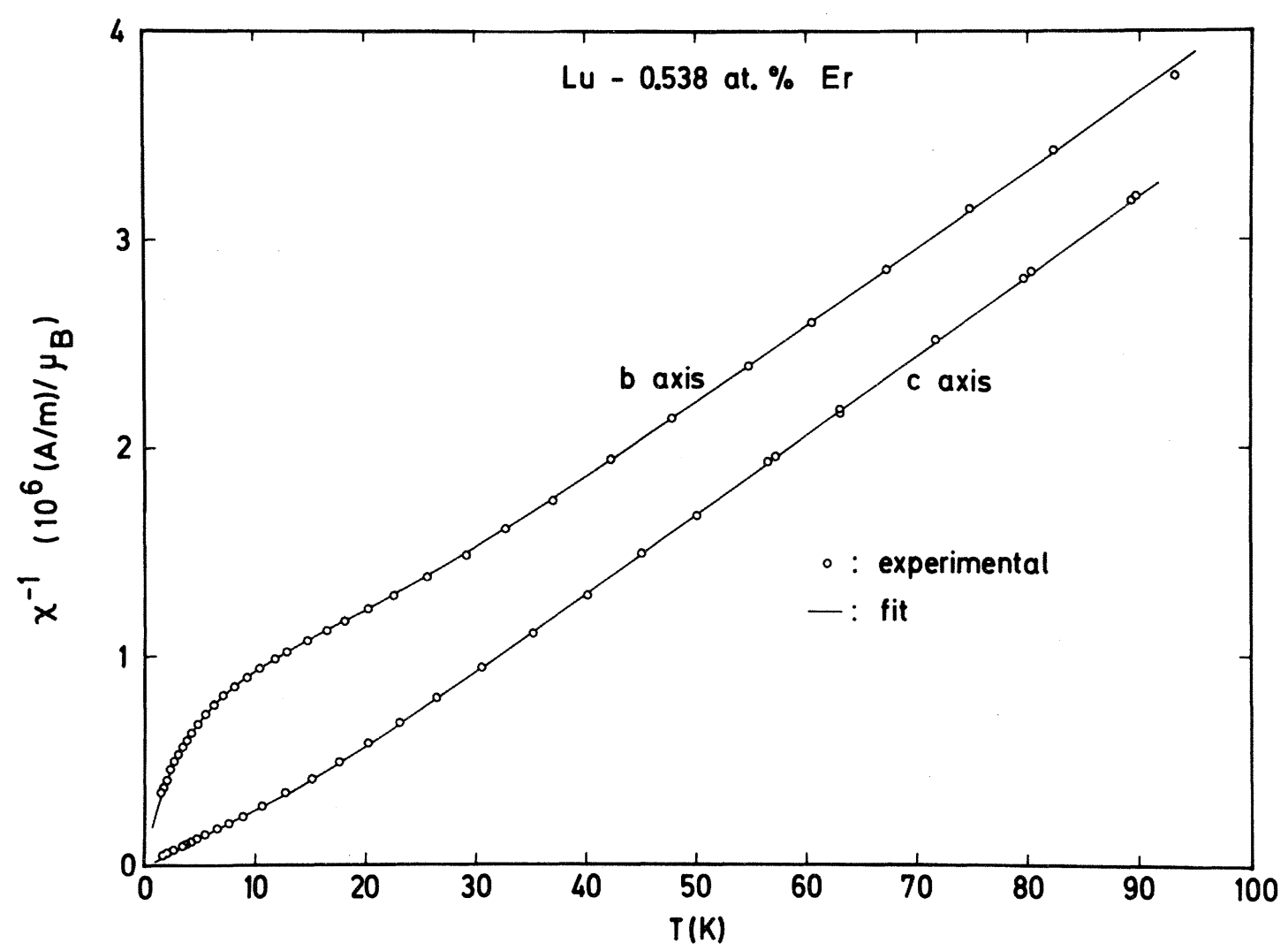

FIG. 11. Reciprocal susceptibility for $\mathrm{Lu}-0.538$ at. $\% \operatorname{Er}\left(H=1.35 \times 10^{5} \mathrm{~A} / \mathrm{m}\right)$. The solid curves are theoretical fits.

tor $\mathrm{s}^{10}$ for both the $\mathrm{Lu}-R$ and the $\mathrm{Y}-R$ systems. These parameters are the multipole amplitudes around a lattice site of the crystal-field potential for a $4 f$ electron. Except for a small monotonic variation due to different $4 f$ radii, no variations with the solute are expected according to simple theories, such as the point-charge model, in which the crystal field is assumed to arise entire-

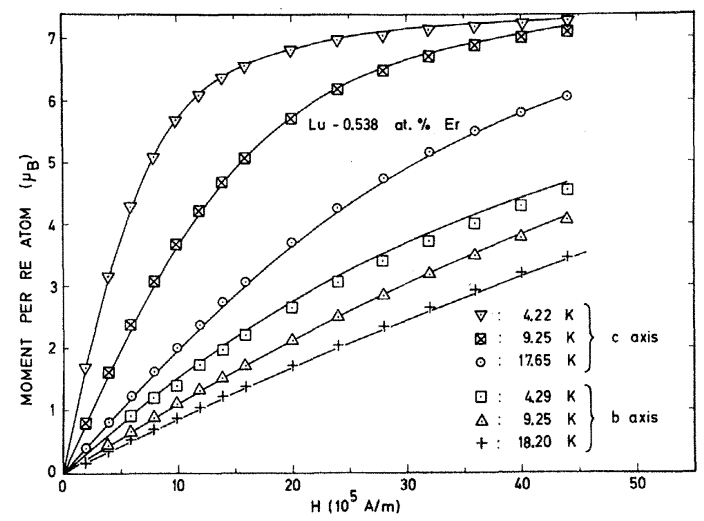

FIG. 12. Isotherms for $\mathrm{Lu}-0.538$ at.\% Er. The solid curves are calculated. ly from the surroundings of the solute ion.

In order to obtain estimates of the crystal-field parameters for the pure-rare-earth metals we compare the results for the $\mathrm{Y}$ and Lu hosts. The parameters $B_{40}, B_{60}$, and $B_{66}$ are within experimental error equal for $\mathrm{Lu}$ and $\mathrm{Y}$. We may expect to obtain different values of $B_{20}$ in $\mathrm{Y}$ and $\mathrm{Lu}$,

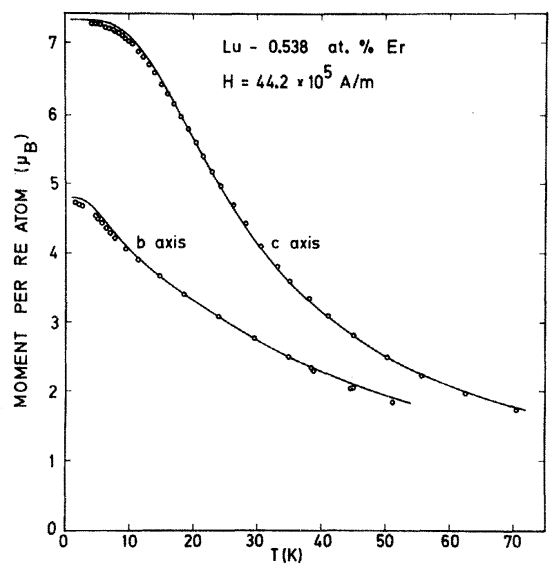

FIG. 13. Isofield magnetization curves for $\mathrm{Lu}-0.538$ at.\% Er. The solid curves are calculated. 
TABLE II. Values of fitted parameters for $\mathrm{Lu}-R$.

\begin{tabular}{cccccc}
\hline \hline Parameter & Units & Lu-0.634-at.\% Tb & Lu-0.481-at.\% Dy & Lu-0.978-at.\% Ho & Lu-0.538-at.\% Er \\
\hline$B_{20}$ & $10^{-1} \mathrm{~K}$ & $+6.5 \pm 10 \%$ & $+1.4 \pm 10 \%$ & $+0.81 \pm 10 \%$ & $-1.6 \pm 8 \%$ \\
$B_{40}$ & $10^{-4} \mathrm{~K}$ & $+6.3 \pm 20 \%$ & $-28 . \pm 10 \%$ & $-0.26 \pm 50 \%$ & $+3.5 \pm 25 \%$ \\
$B_{60}$ & $10^{-5} \mathrm{~K}$ & $-1.3 \pm 20 \%$ & $+3.4 \pm 10 \%$ & $-1.7 \pm 10 \%$ & $+3.1 \pm 5 \%$ \\
$B_{66}$ & $10^{-4} \mathrm{~K}$ & $+2.0 \pm 40 \%$ & $-3.4 \pm 10 \%$ & $(+) 1.6 \pm 10 \%$ & $-3.2 \pm 3 \%$ \\
$\lambda_{\perp}$ & $10^{5}(\mathrm{~A} / \mathrm{m}) /\left(\mu_{B} /\right.$ atom $)$ & $+0.1 \pm 50 \%$ & $+0.55 \pm 20 \%$ & $+0.05 \pm 50 \%$ & $-0.20 \pm 70 \%$ \\
$\lambda_{\|}$ & $\cdots$ & $-4.5 \pm 20 \%$ & $-4.3 \pm 10 \%$ & $-0.56 \pm 30 \%$ & $+0.14 \pm 25 \%$ \\
\hline \hline
\end{tabular}

because the point-charge contribution to $B_{20}$ from the nearest-neighbor ions is proportional to $(c / a$ $\left.-\sqrt{\frac{8}{3}}\right)$. This parameter takes the values 0.060 and

TABLE III. Crystal-field parameters divided by corresponding Stevens factors. The parameters in $\mathrm{Y}$ are obtained from Refs. 1 and 2. The values are in $\mathrm{K}$.

\begin{tabular}{lcccr}
\hline \hline Lu host & Tb & Dy & Ho & \multicolumn{1}{c}{ Er } \\
\hline$B_{20} / \alpha$ & $-63 \pm 6$ & $-22 \pm 2$ & $-36 \pm 4$ & $-63 \pm 5$ \\
$B_{40} / \beta$ & $5.2 \pm 1$ & $48 \pm 5$ & $0.8 \pm 0.4$ & $8 \pm 2$ \\
$B_{60} / \gamma$ & $12 \pm 2$ & $33 \pm 3$ & $13 \pm 1$ & $15 \pm 1$ \\
$B_{66} / \gamma$ & $-179 \pm 72$ & $-327 \pm 33$ & $(-) 123 \pm 12$ & $-156 \pm 5$ \\
Y host & & & & \\
$B_{20} / \alpha$ & $-94 \pm 9$ & $-53 \pm 3$ & $\ldots$ & $-122 \pm 12$ \\
$B_{40} / \beta$ & $4.8 \pm 1$ & $44 \pm 4$ & $\ldots$ & $13.5 \pm 6$ \\
$B_{60} / \gamma$ & $13 \pm 4$ & $32 \pm 3$ & $\ldots$ & $12 \pm 1$ \\
$B_{66} / \gamma$ & $-89 \pm 45$ & $-323 \pm 32$ & $\cdots$ & $-134 \pm 13$ \\
\hline \hline
\end{tabular}

0.049 in $\mathrm{Y}$ and $\mathrm{Lu}$, respectively. The variation of $B_{20}$ into the $c / a$ ratio obtained from high-temperature paramagnetic magnetization measurements ${ }^{13}$ predicts a difference of $B_{20}$ for corresponding $\mathrm{Y}$ and Lu alloys of the same order of magnitude as observed here. These results therefore indicate that, except for differences in lattice constants, the crystal fields in $\mathrm{Y}$ and $\mathrm{Lu}$ are probably representative of the crystal fields in the pure-rareearth metals.

\section{ACKNOWLEDGMENTS}

The authors wish to thank Professor V. Frank and Professor A. R. Mackintosh for many fruitful discussions and for their continued interest. The authors also gratefully acknowledge the care taken by Research Chemicals to provide the pure lutetium material necessary for this research.
*Present address: Department of Physics, University of Odense, DK-5000 Odense, Denmark.

${ }^{1} \mathrm{~J}$. Hog and P. Touborg, Phys. Rev. B $\underline{9}, 2920$ (1974).

${ }^{2} \mathrm{~J}$. Hog and P. Touborg, Phys. Rev. B 11 , 520 (1975).

${ }^{3}$ P. Touborg, J. Hog, G. J. Cock, and L. W. Roeland, Phys. Rev. B 10, 2952 (1974).

${ }^{4} \mathrm{O}$. Rathmann, J. Als-Nielsen, P. Bak, J. Hog, and P. Touborg, Phys. Rev. B 10, 3983 (1974).

${ }^{5}$ A. J. Freeman, in Magnetic Properties of the Rare Earth Metals, edited by R. J. Elliott (Plenum, New York, 1972).

${ }^{6} \mathrm{~J}$. Jensen, J. G. Houmann, and H. Bjerrum Møller (unpublished); R. M. Nicklow, N. Wakabayashi, M. K. Wilkinson, and R. E. Reed, Phys. Rev. Lett. 27, 334 (1971).
${ }^{7}$ O. Jepsen and O. K. Andersen (private communications). ${ }^{8} \mathrm{~K}$. A. McEwen and P. Touborg, J. Phys. F $\underline{3}, 1903$ (1973).

${ }^{9}$ O. V. Nielsen and V. I. Zaitzev, J. Phys. E $\underline{6}, 1022$ (1973).

${ }^{10}$ A. Abragam and B. Bleaney, Electron Paramagnetic Resonance of Transition Ions (Clarendon, Oxford, 1970).

${ }^{11} \mathrm{O}$. Rathmann (private communication).

${ }^{12}$ P. H. Bly, W. D. Corner, and K. N. R. Taylor, J. Appl. Phys. 40, 4787 (1969).

${ }^{13}$ R. Aleonard, P. Boutron, and D. Bloch, J. Phys. Chem. Solids 30, 2277 (1969); P. Touborg, S. Balling, and K. M. Ravn (unpublished). 